\title{
Capture Cross Section Evaluations of 18 Stable Fission Products
}

\author{
Dr. Maher N.Sarsam ${ }^{1}$, Dr. Bashair Mohammed Saied ${ }^{2}$, Dr. Inam Noori Ibrahim ${ }^{3}$ \\ ${ }^{1}$ Alsalam Univerity Collge,Iraq , ${ }^{2}$ Baghdad University-College of education Ibn Al-Haitham,Iraq ${ }^{3}$ Ministry of \\ education, Iraq
}

\begin{abstract}
A comparison of the capture cross sections in the energy range of 0.1-20 MeV of some 18 stable fission products using the main international libraries have been calculated. A weighted average values for the 18 isotopes was found. Curves of all considered data are presented.

Key words: Stable fission products, ${ }^{109 \mathrm{Ag}},{ }^{81} \mathrm{Br}-81,{ }^{111} \mathrm{Cd},{ }^{140} \mathrm{Ce},{ }^{142} \mathrm{Ce},{ }^{133} \mathrm{Cs},{ }^{155} \mathrm{Gd},{ }^{143} \mathrm{Nd},{ }^{145} \mathrm{Nd},{ }^{134} \mathrm{Xe},{ }^{146} \mathrm{Nd}$, ${ }^{108} \mathrm{Pd},{ }^{141} \mathrm{Pr},{ }^{101} \mathrm{Ru},{ }^{94} \mathrm{Zr},{ }^{154} \mathrm{Sm},{ }^{89} \mathrm{Y},{ }^{92} \mathrm{Zr}$, weighted average
\end{abstract}

\section{Introduction}

The fission in a nuclear reactor produces hundreds of different products of radionuclide having neutron to proton ratio of over 1.5 , these unstable radio nuclides decays toward stability mainly by a series of beta emission ${ }^{[1]}$. Some of the fission fragments are nuclides having a large cross sections for neutron capture, even in a small quantities and/or small half life, they can remove from the reactor a number of neutrons which, otherwise, could be contribute to the chain reaction ${ }^{[2]}$. Evaluation of neutron capture cross sections is one of the most important subject needed to the save work of reactors ${ }^{[3]}$. The fission products are needed too in other fields of calculations such as understanding the nature of fission process, determination of the fuel burn- up, performing shielding calculation, calculation of decay heating power, estimation of the amount of gas production, nuclear transmutation of fuel in different types of nuclear reactors, estimation of radiation damage of all reactor material and components, neutron dosimetry of produced nuclides and other special applications ${ }^{[4]}$. A great effort has been devoted to the cross section evaluation of major isotopes pertinent to reactor application including particularly the Uranium and Plutonium isotopes, however, the effort to evaluate fission product data are less than the effort given for the actinide data. The nuclear fission of a fissile material is a complicated process in which more than 500 different products of radio nuclides of about 40 elements of the periodic table (ranged from $Z=31$ to 68 ) are produced.Fission products belonging to about 90 important mass chains ${ }^{[5]}$, they are ranging from Nickel to Erbium. Only about $25 \%$ of them are creating in both ground and isomeric states. Besides this, but with a very little probability, another contribution comes from light fission products (tritium, helium) generated in the ternary nuclear fission.

Most of these fission products may be formed in different ways, as a primary event or as they have extra neutrons, they tend to decay to more stable isotopes through beta emission constituting the fission chains. Each fission chain is formed of a certain number of fission products which are, in general, radioactive such as:[6 ]

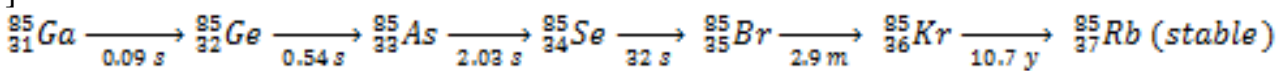

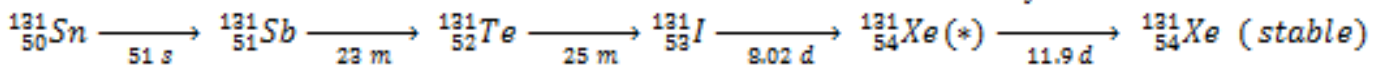

A number of different institutions have been engaged in considerable theoretical and experimental efforts to build their national nuclear data libraries. We select 18 of the stable fission products produced in LWR which represent $34 \%$ of the total of fission products yield, this number is determined in the base of their yields, half-life and in particularly their capture cross sections. The most recent evaluated neutron nuclear data libraries concerning the fission products are actually JENDL- $3^{[7]}$ and JENDL- $4^{[8]}$ from Japan, ENDF/B-V1 release 8 from United States ${ }^{[9]}$, JEFF-3.0 and JEF-2.2 from Europe ${ }^{[10]}$, BROND-2 from Russia ${ }^{[11]}$ and CENDL-3.0 from China ${ }^{[12]}$. These libraries are considered now as containing the most up-to-date nuclear data of evaluated (recommended) cross sections, spectra, angular distributions, fission product yields, thermal neutron scattering, photo-atomic reactions, and other data which are important in neutron-induced reactions relevant to reactor calculations ${ }^{[13,14]}$. They contain too cross sections of numbers of fission products nuclides according to their yields, half-lives, capture, elastic, inelastic and absorption cross sections. In spite of all these voluminous and important data the International Atomic Energy Agency (IAEA), point out that, there are still considerable discrepancies among the evaluated data sets ${ }^{[15]}$. The data of some of the isotopes given by the libraries were obtained by a special numerical evaluation method based on the fitting of nuclear fragments of mass distributions by several Gaussian functions ${ }^{[16,17]}$. The generation of nuclear data based on theoretical physic models is frequently used when no experimental data are available ${ }^{[18]}$. In particular the total cross sections above the resonance region are generally evaluated using the optical model by fitting the data to the measured 
total cross sections. This method allows filling the gaps in the data of experimental results.These libraries contains all kinds of nuclear information principally the capture cross sections of fission nuclides which accounts for more than $40 \%$ of the total neutron absorption cross section of materials constituting the reactor core $^{[19]}$, and it contribute approximately to about $9 \%$ of the reactor reactivity (as their values are proportional to the fuel burn-up or the neutron flux). Using a weighted average formula, we obtain an average value for each of the isotopes. Results of the calculated capture cross sections in reduced energy intervals with the original data are presented in figures.

\section{Results}

The capture cross section of twenty stable fission products (Ag-109, Br-81, Cd-111, Ce-140, Ce-142, Cs-133, Gd-155, Nd-143, Nd-145, Xe-134, Nd-146, Pd-108, Pr-141, Ru-101, Zr-94, Sm-154, Y-89, Zr-92), are plotted using the published data of the main libraries, and an average evaluated value for each isotope is calculated using the following weighted mean formula ${ }^{[20]}$,

$\mathrm{Y}=\frac{\sum w_{i} y_{i}}{\sum w_{i}}$

Where: $\quad w_{i}=1 / \sigma_{i}^{2}$ and $\sigma_{i}=$ standard deviation of sample $\mathrm{i}$

Some libraries declare the uncertainty of the capture cross section values which we considered, otherwise we used $10 \%$ for the unmentioned error.

\section{Conclusion}

Figures (1-18) shows the capture cross sections of eighteen stable fission products. The evaluations are based on the main international data libraries using an averaging formula. The cross sections are probabilistic events, two important types of experimental errors known as systematic and random errors exist, thus there is always some uncertainty in all measurements. It's clear that the average weighted values eliminate a large deviation of values or of errors. This method is very important for some problems such as neutron dosimetry, fuel burn-up and determination of isotope production.

Fig. 2: Capture Cross Section of $\mathrm{Br}-81$

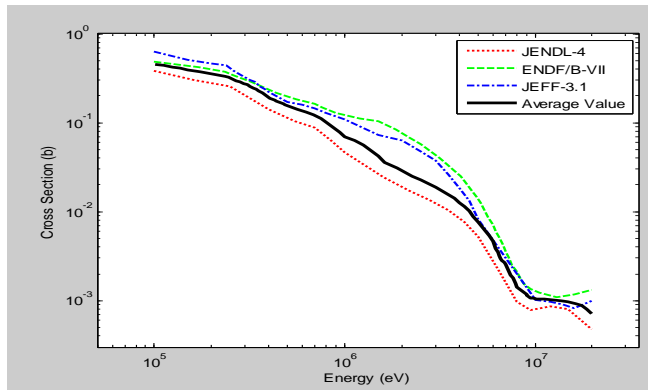

Fig. 4 : Capture Cross Section of Ce-140
Fig. 1: Capture Cross Section of Ag- 109

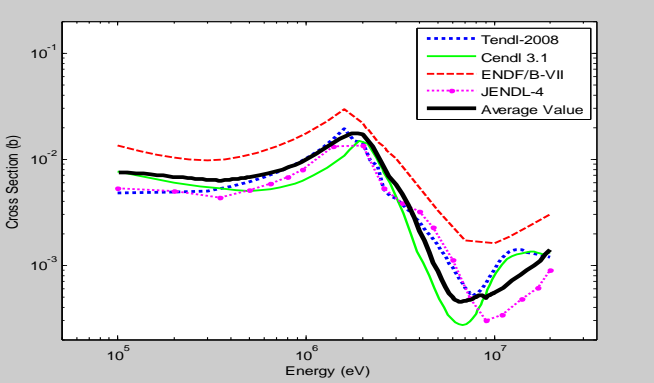

Fig. 3 : Capture Cross Section of Cd-111

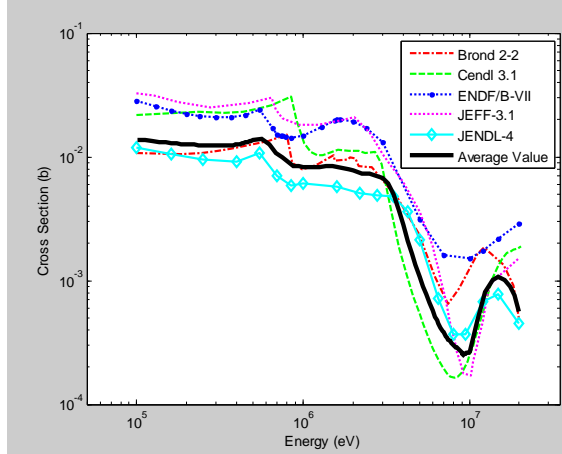

Fig 6 : Capture Cross Section of Cs-133

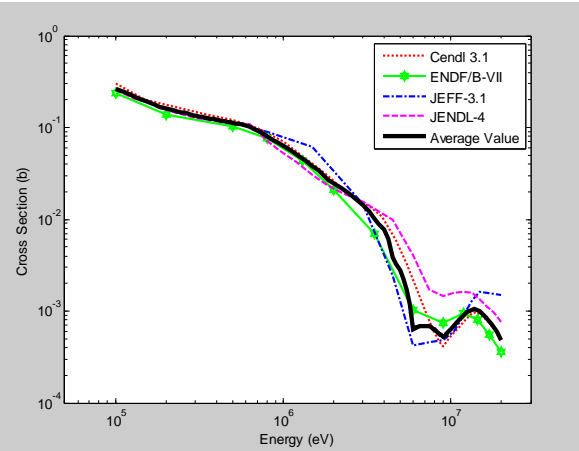

Fig 5 : Capture Cross Section of Ce-142 


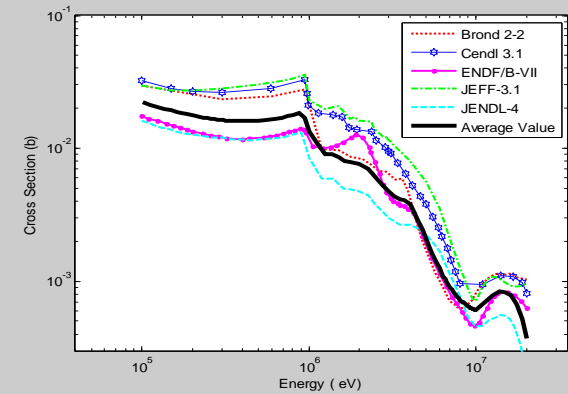

Fig 8: Capture Cross Section of Gd-155

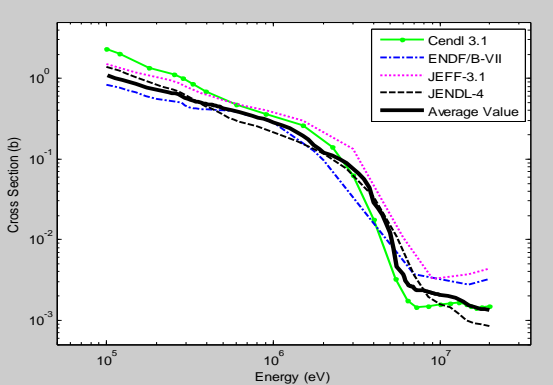

Fig 7: Capture Cross Section of Zr-92

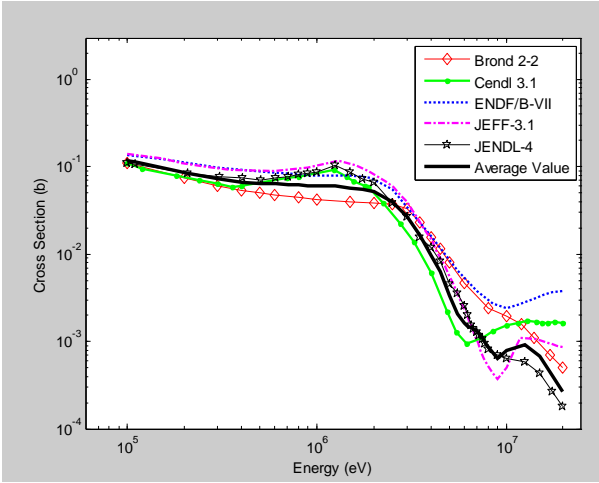

Fig 10: Capture Cross Section of Nd-145

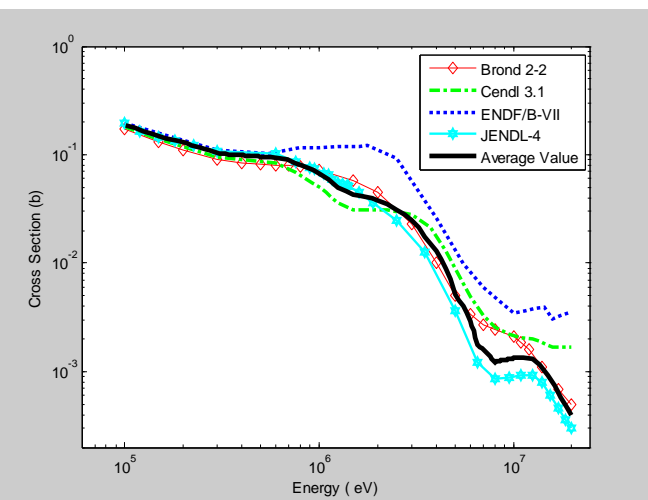

Fig 9: Capture Cross Section of Nd-143

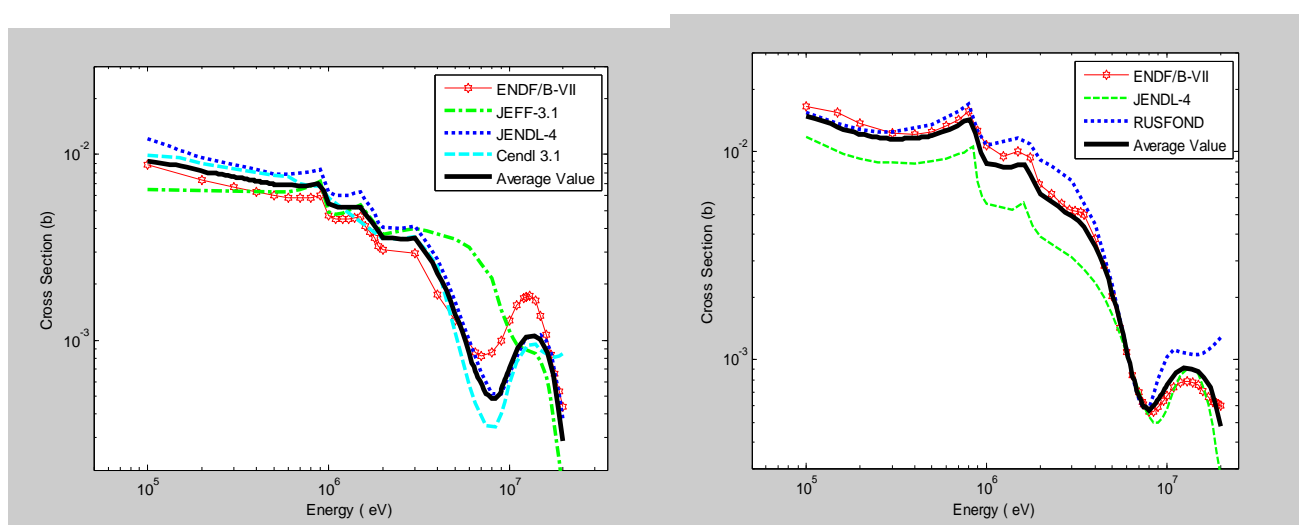

Fig 12: Capture Cross Section of Xe-134

Fig 11: Capture Cross Section of Y-89

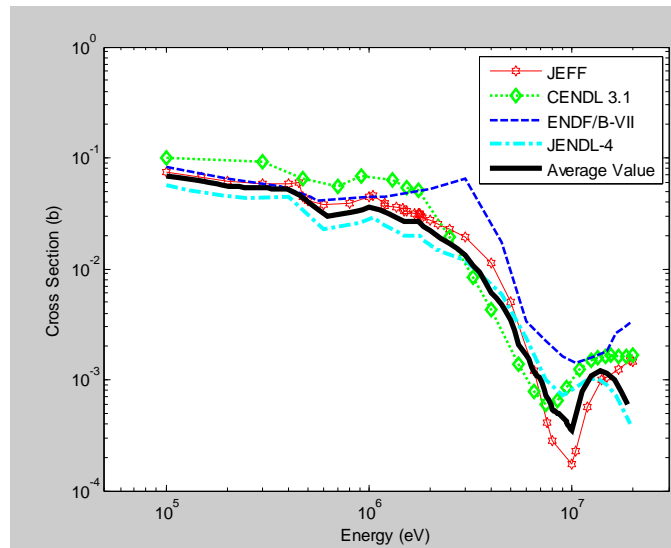

Fig 14: Capture Cross Section of Pd-108

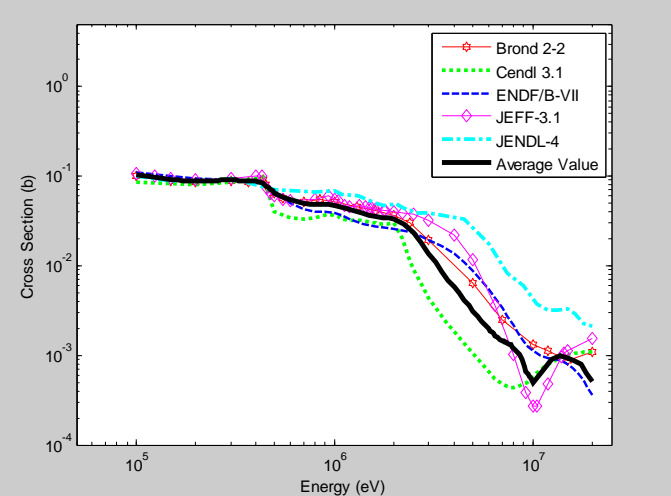

Fig 13: Capture Cross Section of Nd-146 


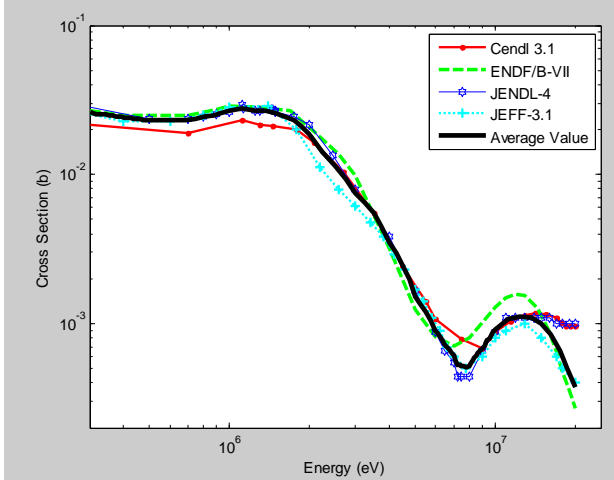

Fig 16 : Capture Cross Section of Ru-101

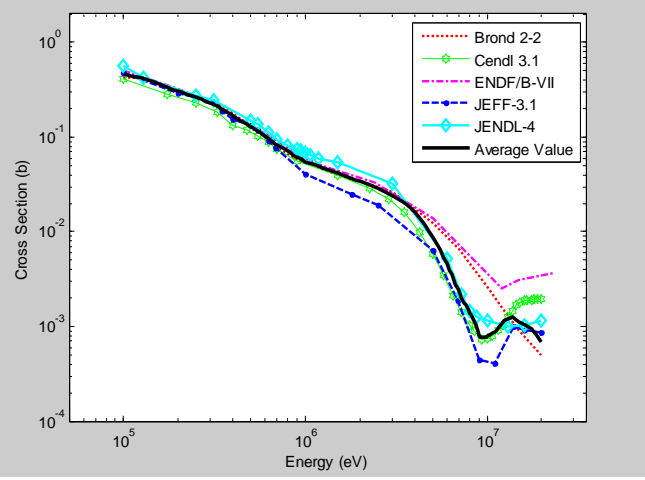

Fig 15 : Capture Cross Section of Pr-141

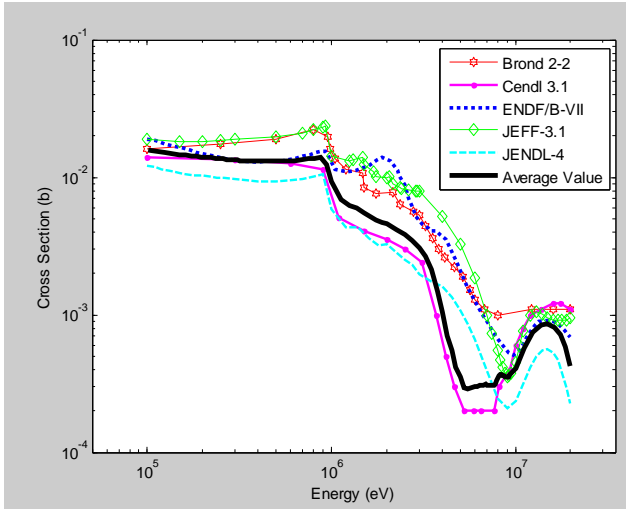

Fig 18: Capture Cross Section of Sm-145

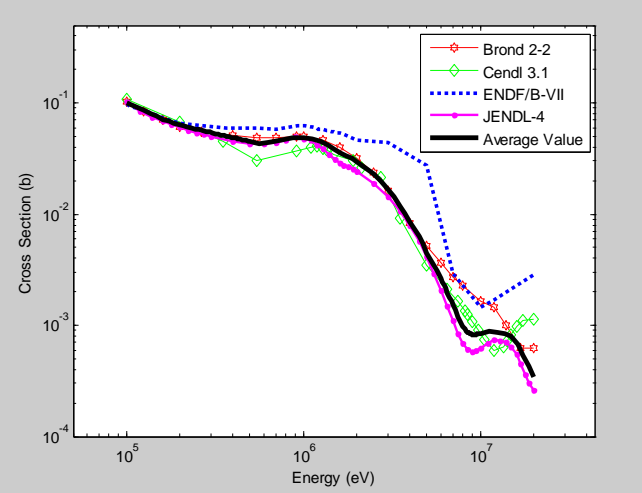

Fig 17 : Capture Cross Section of Zr-94

\section{References}

F.H. Attix” Introduction to Radiological Physics and Radiation Dosimetry “.John Wiley \& sons Inc. 2007. James J. Duderstadt and Louis J. Hamilton” Nuclear Reactor Analysis “.John Wiley \& sons Inc. 1975.

JNDC Nuclear Data Library of Fission Products". K.Tasaka, H. Ihara, M. Akiyama, T. Yoshide, Z.I.Matumoto and R Nakasima. JAERI 1287 june 1983

Fission product nuclear data “. International Atomic Energy Agency, NEA/NSC/DOC 92-9. 5- James E. Martin,” physics for radiation production"

Wiley-VCH Company 2006,(ISBN 9783527406111)

Radioactive Decays at Limits of Nuclear Stability ". M.Pfutzner, M.Karry, L.V.Grigorenko and N.Riisager. arXiv:1111.0482V1 . Nov. 2011

JENDL (Japanese Evaluated Nuclear Data Library) "J.Nucl.Sci.Technol.39, 1125(2002)

K.Shibata etal " JENDL - 4.0 A New Library for Innovative Nuclear Energy Systems."

Conference on Nuclear Data for Science and Technology. April 26-30,2010 Jeju Island, Korea

M.Herman and A.Ttkov “ ENDF-6 Evaluated Nuclear data files (Format Manuel)"BNL-90365 , Brokhaven National Laboratories (April 2009)

JEFF-3.1.1 Nuclear Data Library” ISBN 978-92-64-99074-6 (OECD 2009)

BROND-2A.I.Blokhin etal"Current Status of Russian Evaluated Neutron Data Libraries “,Proc.International Conf.onNuclearDataForScienceAndTechnology ,Gatlinburg,Tennessee,USA,May 9-13,1994,Vol.2,P.695(1994)

ENDL-3 “Zhuang, Liu and Zhang” Chinese Evaluated Nuclear Data Library Journal of Nuclear Science and Technology (2002) M.Lammer and A.L.Nichols " $3^{\text {rd }}$ International Workshop On Nuclear Fission and Fission Products Spectroscopy". (2005), doi:10.1063/1.2137259

B.Pritychenko, S.F.Mughaghab and A.A.Sonzogni "Calculation of Maxwellian-Averaged Cross Sections and Astrophysical Reaction Rates Using ENDF/B-vii-0, JEFF-3,1, JENDL 3.3 and ENDF/B-VI.8 Evaluated Nuclear Reaction Data Libraries" Report BNL-82224-2009-JA (2009

IAEA Coordinated Research Project On Fission Product Yields Data For Minor Actinides" AIP Conf.Proc. November 72005 volume 798 99. 285-296

B.R.Rider and T.R.England "Fission Product Yield Evaluation for the U.S.A Evaluated Nuclear Data Files.LA-UR-94-3318 EXFOR "Exchange Formats ManualIAEA Nuclear Data Section, 2008.”, IAEA-NDS-207.

Debra Ann Rutherford "Theoretical and Experimental Cross Sections for Neutron Reactions on Zinc - 64". LA-11233T (1988) IAEA Coordinated Research Project On Fission Product Yield Data For Minor Actinides Up To 150 MeV AIP Conf. Proc. 798 , pp. 285-296

M.F.James, R.W.MillsandD.R.Weaver," The Use of the Normalized Residual In Using Experimental Data and In Treating Outliers."Rep.AEA-RS-1082 UKAEA (1991),Nucl.Instr.Meth. A313(1992)277

T.R.England and B.F.Rider," Evaluation and Compilation of Fission Product Yields" LA-UR-94-3106, ENDF-349 